\title{
Idiopathic malabsorption due to bile acid synthesis defects
}

INSERM

\section{Source}

INSERM. (1999). Orphanet: an online rare disease and orphan drug data base. Idiopathic malabsorption due to bile acid synthesis defects. ORPHA:84065

Idiopathic malabsorption due to increased acid bile synthesis is an intestinal disease of unknown etiology characterized by an overproduction of bile acids which leads to chronic watery diarrhea. 